\title{
LAMPOON techniques to prevent or manage left ventricular outflow tract obstruction in transcatheter mitral valve replacement
}

\author{
Brian C. Case ${ }^{1}$, John C. Lisko², Vasilis C. Babaliaros ${ }^{2}$, Adam B. Greenbaum ${ }^{2}$, Lowell Satler ${ }^{1}$, \\ Itsik Ben-Dor ${ }^{1}$, Brian J. Forrestal ${ }^{1}$, Charan Yerasi ${ }^{1}$, Norihiko Kamioka ${ }^{2}$, Toby Rogers ${ }^{1,3}$, Ron Waksman ${ }^{1}$, \\ Robert J. Lederman ${ }^{3}$, Jaffar M. Khan ${ }^{3}$ \\ ${ }^{1}$ Section of Interventional Cardiology, MedStar Washington Hospital Center, Washington, DC, USA; ${ }^{2}$ Structural Heart and Valve Center, Emory \\ University Hospital, Atlanta, GA, USA; ${ }^{3}$ Cardiovascular Branch, Division of Intramural Research, National Heart, Lung and Blood Institute, \\ National Institutes of Health, Bethesda, MD, USA \\ Correspondence to: Jaffar M. Khan, BM, BCh. Cardiovascular Branch, Division of Intramural Research, National Heart, Lung and Blood Institute, \\ National Institutes of Health, Bethesda, MD, USA. Email: jaffar.khan@nih.gov.
}

\begin{abstract}
Transcatheter mitral valve replacement (TMVR) is a therapeutic option for patients with severe mitral disease who are deemed inoperable due to their overall surgical risk. The close relationships between the left ventricular outflow tract (LVOT) and the anterior mitral valve leaflet can lead to LVOT obstruction, a common complication with high mortality. Predicting and preventing LVOT obstruction is therefore essential, prior to TMVR. Laceration of the Anterior Mitral leaflet to Prevent Outflow ObtructioN (LAMPOON) is a transcatheter electrosurgical technique to split the anterior mitral valve leaflet immediately prior to TMVR. The technique has been studied in a prospective clinical trial and has evolved with many iterations for specific anatomies. In this review, we discuss the different LAMPOON techniques to prevent and treat LVOT obstruction.
\end{abstract}

Keywords: Transcatheter mitral valve replacement (TMVR); left ventricular outflow tract obstruction (LVOT obstruction); LAMPOON; tip-to-base LAMPOON; antegrade LAMPOON

Submitted Aug 11, 2020. Accepted for publication Dec 22, 2020.

doi: 10.21037/acs-2020-mv-25

View this article at: http://dx.doi.org/10.21037/acs-2020-mv-25

\section{Introduction}

Mitral valve disease is a leading global cause of morbidity and mortality $(1,2)$. Many patients are unsuitable for surgical mitral valve repair or replacement due to a high predicted risk of mortality $(3,4)$. In a subset of high-risk patients with suitable anatomy, transcatheter mitral valve replacement (TMVR) may be a therapeutic option (5). Pending the approval of dedicated devices, transcatheter aortic valves may be implanted in the mitral position in a bioprosthetic valve (valve-in-valve), mitral annuloplasty ring (valve-in-ring) or in native mitral annular calcification (valve-in-MAC) $(6,7)$. In-hospital, thirty-day and one-year mortality rates are highest for valve-in-MAC as compared to valve-in-ring or in valve-in-valve $(6,7)$.

The close relationships between the left ventricular outflow tract (LVOT) and the anterior mitral valve leaflet can lead to LVOT obstruction. The incidence of LVOT obstruction in TMVR occurs in up to $10-40 \%$ of valve-inMAC, $5 \%$ of valve-in-ring, and $0.7-2 \%$ of valve-in-valve cases $(6,7)$. When this phenomenon occurs, outcomes are poor, with in-hospital mortality rates as high as $62 \%(8)$. Furthermore, there is a high percentage screening failure, estimated to be as high as $89 \%$, with the most common exclusion criteria being excessive frailty, severe tricuspid regurgitation, right ventricular dysfunction, prior aortic valve therapy, severe MAC and risk for LVOT obstruction $(9,10)$. The use of a multidisciplinary heart team to assess all these patient and procedural factors are important when deciding when to perform a TMVR procedure. It is important to predict the risk of LVOT obstruction after 
TMVR and to use appropriate techniques to prevent this complication. In this review, we discuss predictors of LVOT obstruction, the use of Laceration of the Anterior Mitral leaflet to Prevent Outflow Obtruction (LAMPOON) to prevent LVOT obstruction, and LAMPOON as a bail-out technique to treat TMVR-induced LVOT obstruction.

\section{LVOT obstruction}

The Mitral Valve Academic Research Consortium defines LVOT obstruction as a sudden decline in hemodynamics following TMVR with intra-procedural transesophageal echocardiographic (TEE) images showing displacement of prosthetic or anterior native mitral valve leaflet obstructing LVOT and an increase in LVOT gradient by $10 \mathrm{mmHg}$ $(11,12)$. However, clinically significant LVOT obstruction is more likely when gradients exceed $30 \mathrm{mmHg}$ (13). Two main mechanisms have been described. "Fixed obstruction" (sometimes referred to as "geometric obstruction") is due to a narrowed and elongated neo-LVOT, caused by the anterior mitral leaflet being pushed towards the interventricular septum from the new transcatheter valve. "Dynamic obstruction" is due to systolic anterior motion (SAM) of the anterior mitral leaflet towards the interventricular septum during systole, due to Bernoulli forces generated by the neo-LVOT (13).

\section{Predicting LVOT obstruction}

"Fixed obstruction" can be predicted by calculating a "neoLVOT" on computed tomography (CT) reconstruction. The neo-LVOT is the smallest cross-sectional area circumscribed by the transcatheter heart valve (THV) and the left ventricular septum, and it also contributes to post-procedural LVOT gradient. The neo-LVOT can be predicted on multi-slice cardiac CT imaging by simulating a virtual THV and measuring the projected minimal crosssectional area (14). Observational studies suggest that a neoLVOT area of less than $170-190 \mathrm{~mm}^{2}$ confers increased risk of LVOT obstruction (11). In addition, the "skirt" neoLVOT can be measured using the area by the THV-covered cells and the interventricular septum, thus simulating the neo-LVOT area if the anterior mitral leaflet was removed surgically or parted by LAMPOON (15).

"Dynamic obstruction" from SAM of the anterior mitral leaflet is harder to predict. Acute aortomitral angulation, reduced mitral annulus-to-interventricular septum (IVS) distance, prominent septal bulge, long anterior mitral leaflet, and redundant mitral chordae are all likely factors that will increase risk. Also, a long anterior mitral leaflet may prolapse back into the THV, interfering with valve closure and ultimately resulting in acute valve failure (14).

\section{LAMPOON}

LAMPOON mimics the surgical technique of resection of the anterior mitral leaflet to prevent LVOT obstruction $(16,17)$. The split anterior mitral leaflet parts away from the LVOT and blood flow, is maintained through the open cells of the THV, despite threatened obstruction were the anterior mitral leaflet intact. In addition, the technique spares the subvalvular apparatus in order to preserve left ventricular function. In the procedure, the anterior mitral valve leaflet is split down the midline using focused radiofrequency energy directed by catheters and guidewires. There are several iterations of the LAMPOON technique, each appropriate in different settings (Central Illustration). The principles of transcatheter electrosurgery, enabling leaflet traversal and precise laceration, have been extensively explored in a recent state-of-the-art review (18). The sections below illustrate these techniques and their appropriate scenarios.

\section{Retrograde "Classic" LAMPOON (Figures 1,2)}

This is this original iteration of the LAMPOON technique $(16,17)$. The procedure steps are illustrated and described in Figures 1,2. The LAMPOON IDE study demonstrated that the LAMPOON procedure was feasible in $100 \%$ of subjects $(n=30)$ across a variety of native and annuloplasty risk anatomies and calcium patterns (13). The in-hospital survival rate was $93 \%$ and thirty-day survival rate was $100 \%$ valve-in-ring and $87 \%$ valve-in-MAC.

There were no strokes in the study. The trial confirmed that LAMPOON is feasible in native and annuloplasty ring anatomies in patients who were otherwise ineligible for treatment, and with acceptable safety (13). The advantage of retrograde LAMPOON is that the vector of laceration aligns along the LVOT. The main disadvantage is the difficulty in positioning the traversal guide underneath the A2-scallop of the anterior mitral valve leaflet. Retrograde LAMPOON is not suitable in patients with mechanical aortic valve bioprostheses, due to the likely hemodynamic instability caused by two guiding catheters across the mechanical aortic valve. 
A

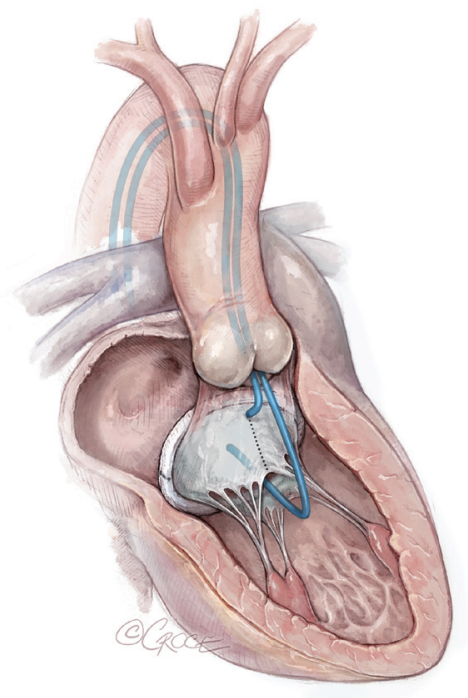

B

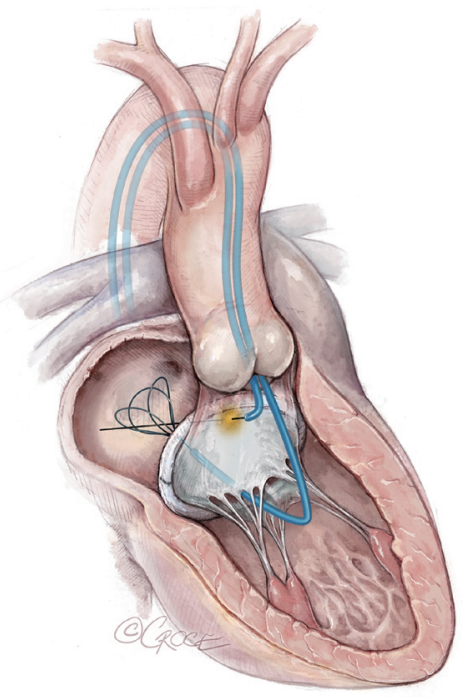

C

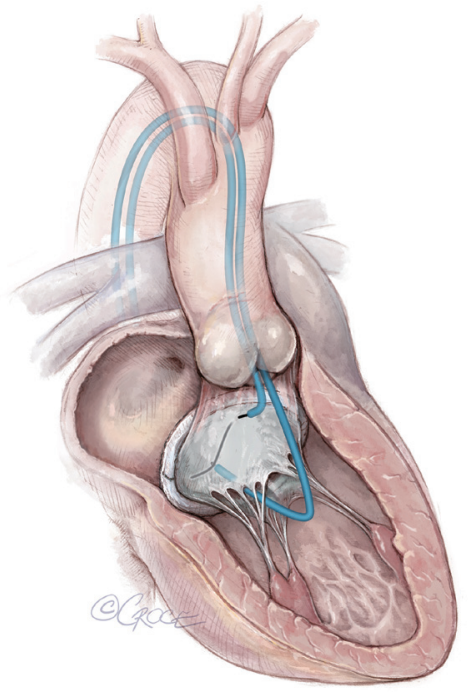

Figure 1 Initial steps in classic, retrograde LAMPOON. (A) Access is via two $6 \mathrm{~F}$ arterial sheaths for LAMPOON and right femoral venous access for TMVR. Two, 6F coronary guiding catheters (JL 3.5), are advanced retrograde across the aortic valve through two femoral sheaths. One guiding catheter is positioned retrograde in the LVOT and abuts the base of the A2 mitral scallop to direct the traversal guidewire, while the other is placed retrograde across the aortic and mitral valves into the left atrium. The left atrial guide catheter is stabilized using a transseptal rail (not depicted). The rail is formed by advancing a balloon tip catheter from a transseptal deflectable catheter (Agilis NxT medium curl, St. Jude Medical, St. Paul, Minnesota) in the left atrium through the main orifice of the mitral valve. The rail is a kinkresistant guidewire (Runthrough NS 0.014-inch, Terumo Interventional Systems, Somerset, New Jersey), and is externalized after snaring. (B) Through the retrograde left atrium catheter, a multiloop snare (Atrieve 18/30, Argon Medical, Plano, Texas) is positioned alongside the rail at the mitral coaptation surfaces. The traversal guidewire is a 0.014-inch $\times 300 \mathrm{~cm}$ guidewire (Astato XS 20, Asahi Intecc USA, Santa Ana, California) inside an insulating polymer jacket wire convertor (Piggyback, Teleflex Vascular Solutions, Minneapolis, Minnesota), inside the retrograde LVOT guiding catheter. The external back end of the guidewire is connected via hemostatic forceps to an electrosurgery pencil and generator (ValleyLab FX, Medtronic Covidien, Minneapolis, Minnesota), set to "pure" cutting mode at $50 \mathrm{~W}$. The traversal guidewire is advanced from the LVOT catheter, penetrating the base of the A2 scallop, during brief ( $<1$ second) electrification into the prepositioned left atrial snare. (C) The guidewire is snared in the left atrium and the transseptal rail is released.

\section{Antegrade LAMPOON (Figure 3)}

The most technically challenging step in retrograde LAMPOON is positioning the retrograde guide catheter at the base of A2. To simplify the procedure, an antegrade approach was developed. The antegrade approach also enables LAMPOON to work in the setting of a mechanical aortic valve. The procedure steps are described in Figure 3. The initial preliminary results $(\mathrm{n}=8)$ demonstrated that the technique was successful in $100 \%$ of patients with a minimal increase in LVOT gradient post-TMVR $\leq 10 \mathrm{mmHg}$. In addition, no patients experienced an insufficient laceration or significant hemodynamic compromise and all patients survived the procedure. The median wire traversal-to-leaflet laceration time was twentysix minutes, median laceration-to-valve implantation time was eighteen minutes and median number of attempts at successful leaflet traversal was one minute. This case series suggests that antegrade LAMPOON is an effective, reproducible and simplified strategy to lacerate the anterior mitral leaflet (AML) prior to TMVR (17). Compared with retrograde LAMPOON, this approach offers greater guide catheter stability and more precise positioning over the target A2 scallop. The main disadvantage is the risk of eccentric mitral valve laceration from the center of A2 medially towards the interatrial septum. A stable pivot provided by the two steerable sheaths is essential to avoid eccentric laceration. 
A

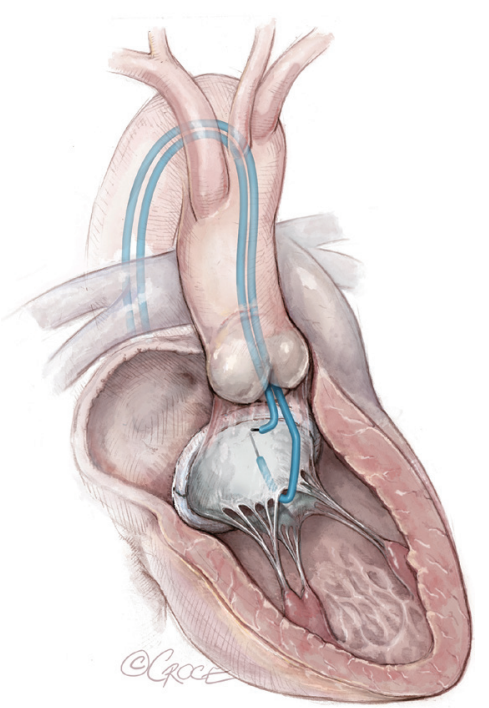

B

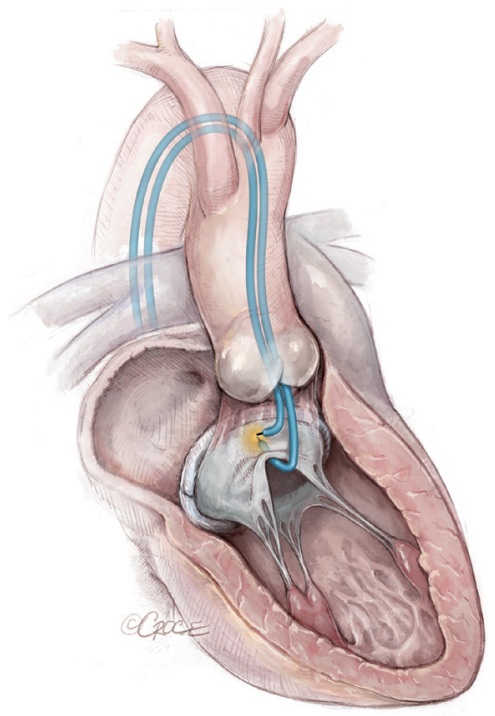

C

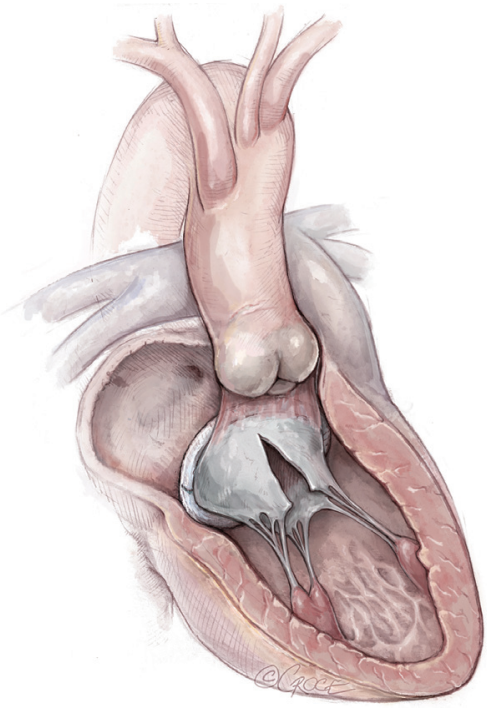

Figure 2 Leaflet laceration, retrograde LAMPOON. (A) A short segment of the Astato guidewire shaft is non-circumferentially focally denuded of its polymer insultation, and then kinked using a scalpel, to form the "flying-V." This modification focuses electrosurgery energy required for laceration on the "inner elbow" of the guidewire. The radiopaque tip of the Piggyback wire converter is locked behind the denuded kinked segment for added fluoroscopic visibility and electrical insulation. The snared tip of the Astato guidewire is then externalized as the kinked segment is advanced till it is positioned across the anterior mitral leaflet. (B) For laceration, tension is applied to both limbs of the guidewire and two guiding catheters during brief (1-2 seconds) electrification at $70 \mathrm{~W}$. Simultaneously, 5\% dextrose is infused through the two catheters to displace blood, preventing char and coagulation, and concentrating charge on the anterior mitral leaflet. (C) Demonstrates midline laceration of the anterior mitral valve leaflet following LAMPOON. Because of the linear electrical laceration, the anterior mitral leaflet maintains some degree of coaptation in diastole, preventing torrential MR prior to TMVR and mitigating the risk of hemodynamic collapse. Following LAMPOON, transseptal TMVR is performed in a standard fashion. TMVR, transcatheter mitral valve replacement; MR, mitral regurgitation.

\section{Tip-to-base LAMPOON (Figure 4)}

When the aorto-mitral curtain is protected by a complete bioprosthetic ring or bioprosthetic mitral valve sewing ring, LAMPOON laceration may be performed from leaflet tip to base, obviating the need for leaflet traversal. This iteration is another major step in simplifying the technique in this subset of patients. These procedure steps are illustrated in Figure 4. For select patients with high-risk anatomy, undergoing $\mathrm{ViV}$ or valve-in-(complete)-ring TMVR, tipto-base LAMPOON appears to be an effective solution to prevent LVOT obstruction (19). The major advantage of tip-to-base LAMPOON is the simplicity afforded by eliminating the traversal step. It is only appropriate in cases where there is an adequate "backstop" to prevent laceration extending into the aorto-mitral curtain and aortic valve. The disadvantages involved include insufficient laceration of the leaflet and inadvertent laceration of adjacent structures, if care is not taken to insulate the active cutting surface adequately.

\section{"Rescue" LAMPOON (Figure 5)}

In cases where there is SAM causing LVOT obstruction after TMVR, LAMPOON can be performed to lacerate the protruding native anterior mitral valve leaflet (20). The technique is similar to when performing "tip-tobase" LAMPOON and is illustrated in Figure 5. This is a useful technique because cases of LVOT obstruction from SAM in the setting of a generous neo-LVOT is difficult to predict, and this treatment can be used as a bail-out and an alternative to alcohol septal ablation. This should not be used as a substitute for improving predictors of dynamic LVOT obstruction. 
A

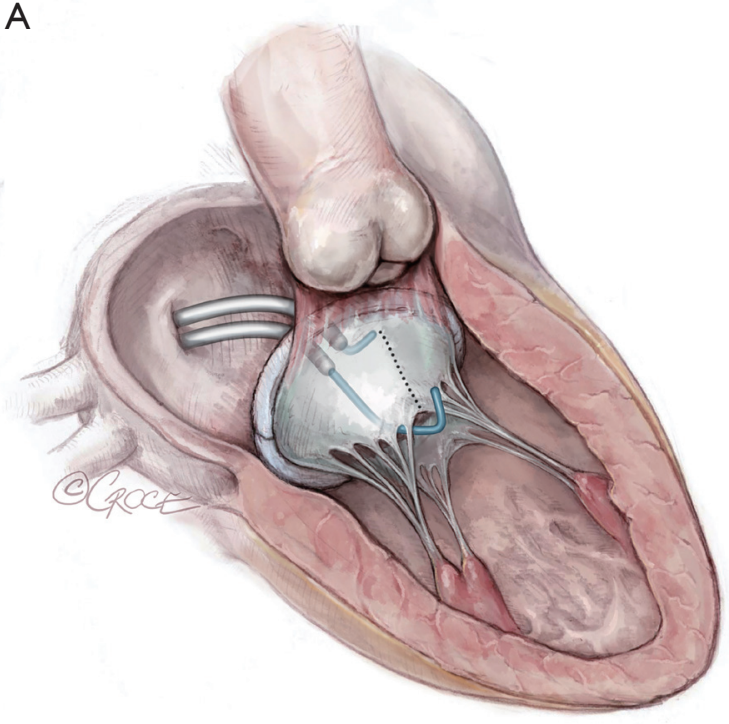

C

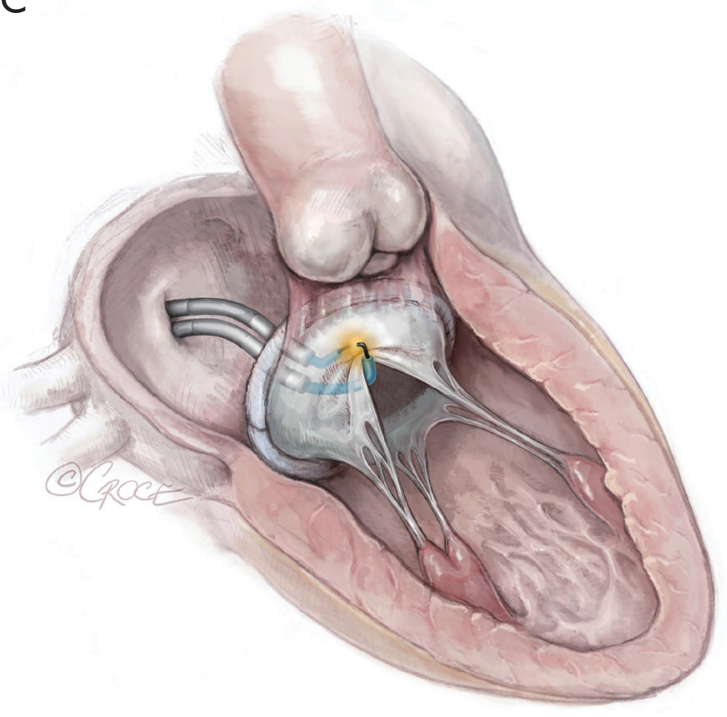

B

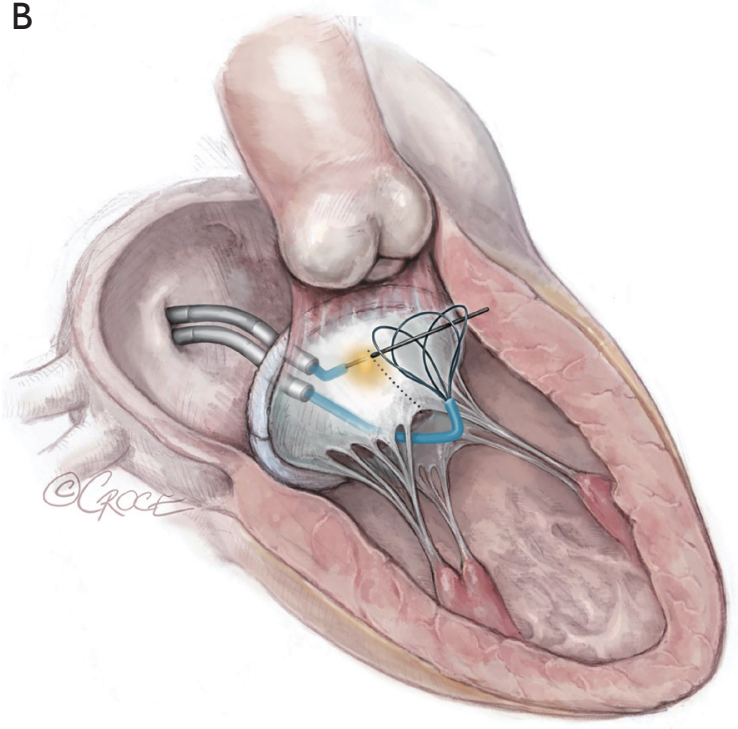

D

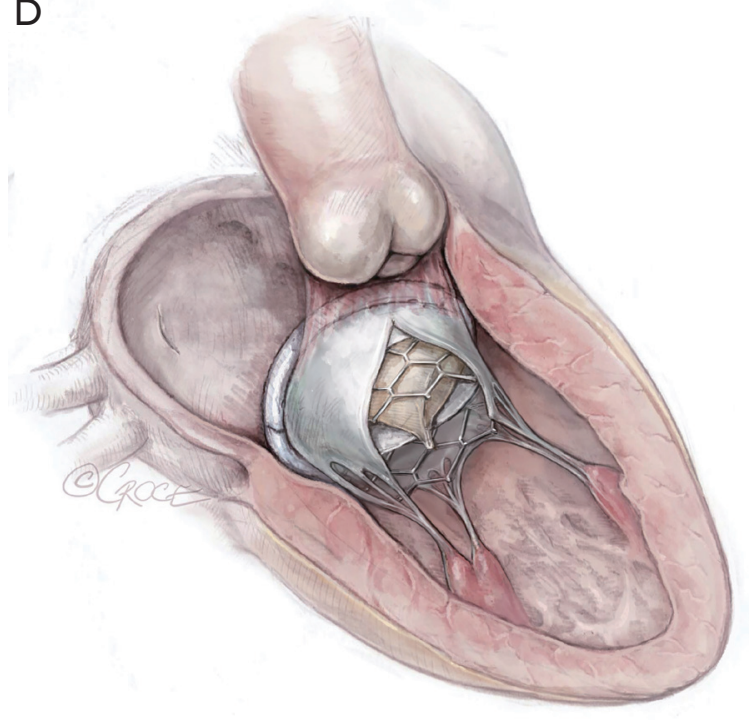

Figure 3 Antegrade LAMPOON. (A) Access is via a $24 \mathrm{Fr}$ DrySeal Flex sheath in the right femoral vein to allow two deflectable sheath through one access site, and subsequently for valve delivery. A $7 \mathrm{~F}$ sheath is placed in a femoral artery for rail guidewire snaring, hemodynamic monitoring, and subsequently for intra-aortic balloon pump positioning (optional). After transseptal puncture, two guidewires are positioned in the left atrium and two deflectable sheaths are placed through the single transseptal puncture. A balloon wedge end-hole catheter is advanced through the major mitral orifice, avoiding chordae, and guided out of the aortic valve. Through this, a rail guidewire (300 cm 0.014" Runthrough guidewire) is delivered and snare-externalized out of the femoral artery (not depicted). The balloon catheter is removed and a JL or EBU guiding catheter is advanced over the rail into the LVOT. (B) A snare (typically 18/30 EnSare or Atrieve) is positioned in the LVOT. A JR4 guiding catheter is advanced through the second steerable sheath to the atrial surface of the base of A2 under TEE guidance. The insulated Astato guidewire is introduced and energized with $50 \mathrm{~W}$ pure cut power and advanced through the anterior mitral leaflet from atrium into LVOT. (C) The Astato guidewire is snared in the LVOT. The "Flying V" is created at the mid-shaft of the guidewire. The "Flying V" is positioned at the anterior mitral leaflet and the two steerable sheaths are aligned with A2 to provide a pivot for centerline laceration. Tension is applied to the LAMPOON guidewire and catheter system to lacerate the anterior mitral leaflet down the centerline with simultaneous $5 \%$ dextrose infusion. (D) TMVR is then performed in the standard fashion, with the leaflet halves splaying away from the LVOT. TEE, transoesophageal echocardiogram; LVOT, left ventricular outflow tract. 


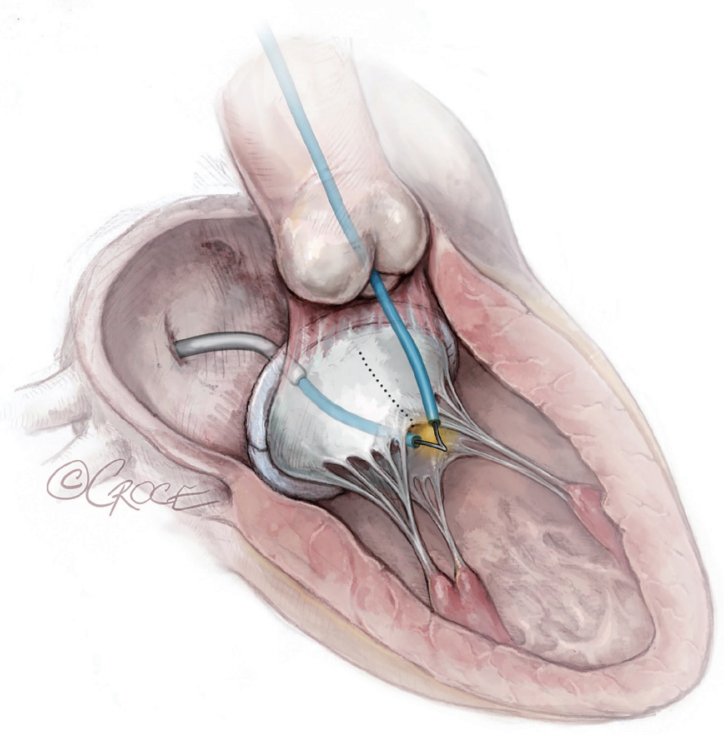

Figure 4 Tip-to-base LAMPOON. Access is via the right femoral vein for steerable sheath and subsequently TMVR. $6 \mathrm{~F}$ arterial access is obtained for snaring. In this modified technique, after transseptal puncture the mitral prosthesis is crossed using a balloon wedge end-hole catheter. An Astato guidewire is advanced through the balloon-wedge catheter and snared in the ascending aorta. The guiding catheter used for snaring is advanced across the aortic valve. The balloon catheter is withdrawn and a guide catheter advanced. The mid-shaft of the Astato guidewire is kinked and positioned at the tip of the anterior bioprosthetic mitral leaflet. The final step is the laceration where the $5 \%$ Dextrose is infused, the wire if electrified at $70 \mathrm{~W}$ pure cut, and slowly pulled back to facilitate laceration. The "Flying V" will reach a hard stop at the valve sewing ring. The LAMPOON system is disassembled, and the deflectable sheath in the left atrium is used to guide a stiff preshaped 0.035 " guidewire into the left ventricle for valve-in-valve TMVR. TMVR, transcatheter mitral valve replacement.

\section{Conclusions}

LVOT obstruction after TMVR continues to be a major concern and carries a high risk of in-patient mortality. LAMPOON is a versatile technique that has been prospectively studied in an early feasibility clinical trial. It enables TMVR in patients who would otherwise be

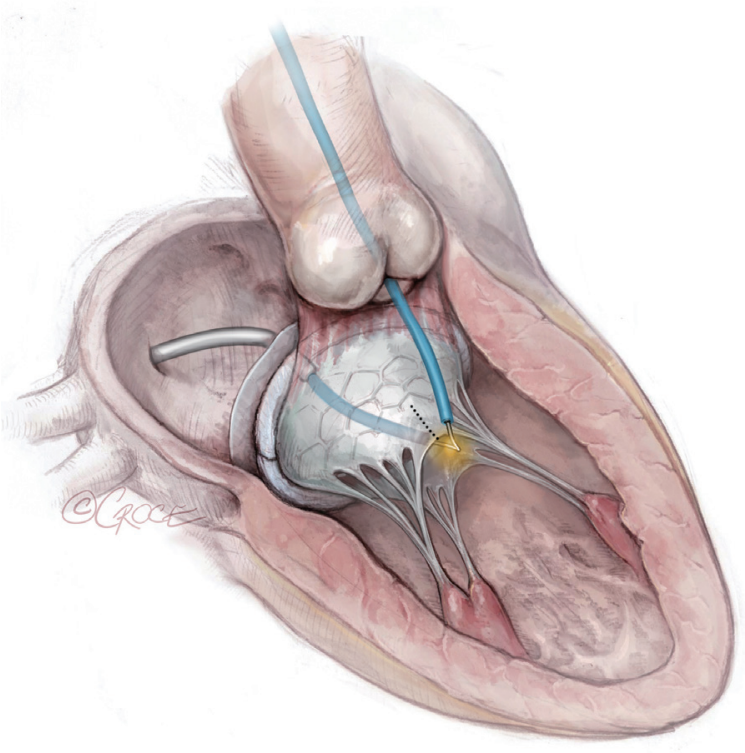

Figure 5 Rescue LAMPOON is an option when there is LVOT obstruction due to SAM after TMVR. Access is via the right femoral vein for an Agilis sheath and femoral artery for a $6 \mathrm{Fr}$ sheath. Following transseptal puncture, a balloon catheter is floated via an Agilis deflectable sheath through the THV. A $300 \mathrm{~cm} \mathrm{0.014"} \mathrm{Astato} \mathrm{XS} 20$ guidewire is advanced through the balloon catheter and snared in the ascending aorta. The balloon catheter is replaced with a JR guide and the "Flying V" is created on the Astato mid-shaft. The Astato guidewire tip is externalized and the "Flying $\mathrm{V}$ " is positioned at the tip of the anterior mitral leaflet between femoral and transseptal guiding catheters. The transseptal guiding catheter is positioned at A2 via a deflectable transseptal sheath under TEE guidance. Tension is applied to both catheters, electrified with $70 \mathrm{~W}$ radiofrequency energy and $5 \%$ dextrose flush and the lacerating edge is pulled upwards from the protruding tip of the anterior mitral leaflet towards the THV frame. The THV frame provides a barrier to prevent deleterious advancement of the laceration edge into the aortomitral curtain and aortic root. The catheters are relaxed and laceration is confirmed on TEE and LVOT gradients are assessed. Electrification can be repeated to achieve effective tip laceration. TMVR, transcatheter mitral valve replacement; LVOT, left ventricular outflow tract; SAM, systolic anterior motion; THV, transcatheter heart valve; TEE, transoesophageal echocardiogram. 
ineligible due to high risk of LVOT obstruction. Iterations of the LAMPOON technique have been developed and may make the procedure easier to perform in certain anatomies.

\section{Acknowledgments}

Funding: Supported by the Division of Intramural Research, National Heart, Lung, and Blood Institute, National Institutes of Health, USA (Z01-HL006040).

\section{Footnote}

Conflicts of Interest: VCB is a consultant for Edwards Lifesciences and for Abbott Vascular, and his employer has research contracts for clinical investigation of transcatheter aortic and mitral devices from Edwards Lifesciences, Abbott Vascular, Medtronic, St Jude Medical, and Boston Scientific. ABG is a proctor for Edwards Lifesciences, Medtronic, and Abbott Vascular. He is a consultant for Transmural Systems. TR is a consultant/proctor for Medtronic and Edwards Lifesciences. He has equity shares in Transmural Systems. RW-Advisory Board: Abbott Vascular, Amgen, Boston Scientific, Medtronic, Philips, Pi-Cardia Ltd., Cardioset; Consultant: Abbott Vascular, Amgen, Biosensors, Biotronik, Boston Scientific, Medtronic, Philips, Pi-Cardia Ltd., Cardioset; Grant Support: Abbott Vascular, AstraZeneca, Biosensors, Biotronik, Boston Scientific, Chiesi; Speakers Bureau: AstraZeneca, Chiesi; Investor: MedAlliance. JMK is a proctor for Edwards Lifesciences and Medtronic. JMK, TR, and RJL are co-inventors on patents, assigned to NIH, on catheter devices to lacerate valve leaflets. NHLBI has a collaborative research and development agreement with Edwards Lifesciences on transcatheter modification of the mitral valve. The other authors have no conflicts of interest to declare.

Open Access Statement: This is an Open Access article distributed in accordance with the Creative Commons Attribution-NonCommercial-NoDerivs 4.0 International License (CC BY-NC-ND 4.0), which permits the noncommercial replication and distribution of the article with the strict proviso that no changes or edits are made and the original work is properly cited (including links to both the formal publication through the relevant DOI and the license). See: https://creativecommons.org/licenses/by-nc-nd/4.0/.

\section{References}

1. Coffey S, Cairns BJ, Iung B. The modern epidemiology of heart valve disease. Heart 2016;102:75-85.

2. Nkomo VT, Gardin JM, Skelton TN, et al. Burden of valvular heart diseases: a population-based study. Lancet 2006;368:1005-11.

3. Mirabel M, Iung B, Baron G, et al. What are the characteristics of patients with severe, symptomatic, mitral regurgitation who are denied surgery? Eur Heart J 2007;28:1358-65.

4. Goel SS, Bajaj N, Aggarwal B, et al. Prevalence and outcomes of unoperated patients with severe symptomatic mitral regurgitation and heart failure: comprehensive analysis to determine the potential role of MitraClip for this unmet need. J Am Coll Cardiol 2014;63:185-6.

5. Regueiro A, Granada JF, Dagenais F, et al. Transcatheter Mitral Valve Replacement: Insights From Early Clinical Experience and Future Challenges. J Am Coll Cardiol 2017;69:2175-92.

6. Yoon SH, Whisenant BK, Bleiziffer S, et al. Outcomes of transcatheter mitral valve replacement for degenerated bioprostheses, failed annuloplasty rings, and mitral annular calcification. Eur Heart J 2019;40:441-51.

7. Guerrero M, Vemulapalli S, Xiang Q, et al. Thirty-Day Outcomes of Transcatheter Mitral Valve Replacement for Degenerated Mitral Bioprostheses (Valve-in-Valve), Failed Surgical Rings (Valve-in-Ring), and Native Valve With Severe Mitral Annular Calcification (Valve-in-Mitral Annular Calcification) in the United States: Data From the Society of Thoracic Surgeons/American College of Cardiology/Transcatheter Valve Therapy Registry. Circ Cardiovasc Interv 2020;13:e008425.

8. Guerrero M, Urena M, Himbert D, et al. 1-Year Outcomes of Transcatheter Mitral Valve Replacement in Patients With Severe Mitral Annular Calcification. J Am Coll Cardiol 2018;71:1841-53.

9. Niikura H, Gossl M, Kshettry V, et al. Causes and Clinical Outcomes of Patients Who Are Ineligible for Transcatheter Mitral Valve Replacement. JACC Cardiovasc Interv 2019;12:196-204.

10. Del Val D, Ferreira-Neto AN, Wintzer-Wehekind J, et al. Early Experience With Transcatheter Mitral Valve Replacement: A Systematic Review. J Am Heart Assoc 2019;8:e13332.

11. Yoon SH, Bleiziffer S, Latib A, et al. Predictors of Left 
Ventricular Outflow Tract Obstruction After Transcatheter Mitral Valve Replacement. JACC Cardiovasc Interv 2019;12:182-93.

12. Stone GW, Adams DH, Abraham WT, et al. Clinical Trial Design Principles and Endpoint Definitions for Transcatheter Mitral Valve Repair and Replacement: Part 2: Endpoint Definitions: A Consensus Document From the Mitral Valve Academic Research Consortium. J Am Coll Cardiol 2015;66:308-21.

13. Khan JM, Babaliaros VC, Greenbaum AB, et al. Anterior Leaflet Laceration to Prevent Ventricular Outflow Tract Obstruction During Transcatheter Mitral Valve Replacement. J Am Coll Cardiol 2019;73:2521-34.

14. Blanke P, Naoum C, Dvir D, et al. Predicting LVOT Obstruction in Transcatheter Mitral Valve Implantation: Concept of the Neo-LVOT. JACC Cardiovasc Imaging 2017;10:482-5.

15. Khan JM, Rogers T, Babaliaros VC, et al. Predicting Left Ventricular Outflow Tract Obstruction Despite Anterior Mitral Leaflet Resection: The "Skirt NeoLVOT". JACC Cardiovasc Imaging 2018;11:1356-9.

Cite this article as: Case BC, Lisko JC, Babaliaros VC, Greenbaum AB, Satler L, Ben-Dor I, Forrestal BJ, Yerasi C, Kamioka N, Rogers T, Waksman R, Lederman RJ, Khan JM. LAMPOON techniques to prevent or manage left ventricular outflow tract obstruction in transcatheter mitral valve replacement. Ann Cardiothorac Surg 2021;10(1):172-179. doi: 10.21037/acs-2020-mv-25
16. Khan JM, Rogers T, Schenke WH, et al. Intentional Laceration of the Anterior Mitral Valve Leaflet to Prevent Left Ventricular Outflow Tract Obstruction During Transcatheter Mitral Valve Replacement: Pre-Clinical Findings. JACC Cardiovasc Interv 2016;9:1835-43.

17. Babaliaros VC, Greenbaum AB, Khan JM, et al. Intentional Percutaneous Laceration of the Anterior Mitral Leaflet to Prevent Outflow Obstruction During Transcatheter Mitral Valve Replacement: First-in-Human Experience. JACC Cardiovasc Interv 2017;10:798-809.

18. Khan JM, Rogers T, Greenbaum AB, et al. Transcatheter Electrosurgery: JACC State-of-the-Art Review. J Am Coll Cardiol 2020;75:1455-70.

19. Case BC, Khan JM, Satler LF, et al. Tip-to-Base LAMPOON to Prevent Left Ventricular Outflow Tract Obstruction in Valve-in-Valve Transcatheter Mitral Valve Replacement. JACC Cardiovasc Interv 2020;13:1126-8.

20. Khan JM, Trivedi U, Gomes A, et al. "Rescue" LAMPOON to Treat Transcatheter Mitral Valve Replacement-Associated Left Ventricular Outflow Tract Obstruction. JACC Cardiovasc Interv 2019;12:1283-4. 